\title{
Fenomenología
}

\section{El concepto del Baby Café como red internacional de apoyo a la lactancia materna}

\section{The Baby Café concept as an international network of support to} breastfeeding

\section{$\mathrm{O}$ conceito do Baby Café como rede internacional de apoio à ama- mentação materna}

\author{
Isabel Baño Piñero ${ }^{1}$, César Carrillo García ${ }^{2}$, Uma Thambidurai ${ }^{3}$, María Emilia Martínez Roche ${ }^{4}$ \\ ${ }^{1}$ Matrona, Máster en Antropología y Doctoranda por la Universidad de Murcia
}

${ }^{2}$ Doctor en Enfermería. Técnico de Formación Sanitaria Especializada. Dirección General de Recursos Humanos del Servicio Murciano de Salud. Universidad de Murcia.

${ }^{3}$ Mother and Child Pte Ltd (Singapur). Matrona, IBCLC $y$ Directora de Mother and Child (Singapur).

${ }^{4}$ Profesora titular de Universidad y Coordinadora de la Unidad Docente de Matronas. Facultad de Enfermería. Universidad de Murcia y Servicio Murciano de Salud

Cómo citar este relato en edición digital: Baño Piñero, I., Carrillo García, C., Thambidurai, U. y Martínez Roche, Ma E. (2015).

El concepto del Baby Café como red internacional de apoyo a la lactancia materna. Cultura de

los Cuidados (Edición digital) 19, 43. Disponible en:< http://dx.doi.org/10.14198/cuid.2015.43.03>

Correspondencia: Facultad de Enfermería. Campus Universitario de Espinardo. Edificio 23. 30100 Murcia.

Correo electronico: isabel.bano1@um.es

Recibido: 11/03//2015; Aceptado: 10/08/2015

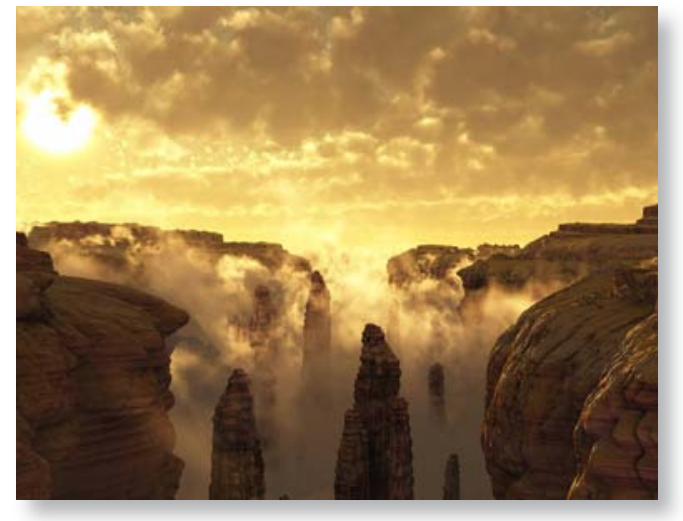

ABSTRACT

The general objective of this work is to reflect on the phenomenon of The Baby Café as a novel and convenient concept in a world of promotion, protection and support of breastfeeding worldwide.

The Baby Café is an initiative to support breastfeeding that coordinates an international network of centers that combines formal support from health professionals who specialize in lactation with informal support groups or networks of breastfeeding.

With the ultimate goal of the promotion, protection and support of breastfeeding, the introduction of The Baby Café initiative encourages more studies of such nature to be conducted, eventually demonstrating the true impact of such initiatives on society; we have developed, through the theoretical framework and a field diary, a qualitative study, which has allowed us to explore the phenomenon of the Baby Cafe in the 10th district of Singapore, during the month of March 2015.

Keywords: Baby Café, Support, International Initiative, Breastfeeding. 


\section{RESUMO}

O objetivo geral deste trabalho consiste em refletir sobre o fenómeno do bebe café como novo e conveniente recurso dentro do mundo da promoção, proteção e apoio a amamentação materna a nível internacional.

O bebe café é uma iniciativa de apoio a amamentação materna que coordena uma rede internacional de centros nos quais se combina o apoio formal proveniente dos profissionais sanitários especializados em amamentação, e no apoio informal próprio dos grupos o redes de apoio a amamentação.

Com o fim de promover, proteger e apoiar a amamentação materna aprofundando a iniciativa do bebe café e incentivando a que se realizem mais estudos que avaliem o verdadeiro impacto que este tipo de iniciativa tem sobre a sociedade; temos desenvolvido, mediante o estado da questão e um diário de campo, um estudo qualitativo, que nos permitiu explorar o fenómeno do bebe café no distrito de Singapura durante o mês de Março 2015.

Palavras chave: Baby Café, Apoio, Iniciativa Internacional, Amamentação Materna.

\section{RESUMEN}

El objetivo general de este trabajo consiste, en reflexionar sobre el fenómeno del Baby Café como recurso novedoso y conveniente dentro del mundo de la promoción, protección y apoyo a la lactancia materna a nivel internacional.

El Baby Café es una iniciativa de apoyo a la lactancia materna, que coordina una red internacional de centros en los que se combina el apoyo formal proveniente de los profesionales sanitarios especializados en lactancia y el apoyo informal propio de los grupos o redes de apoyo a la lactancia.

Con el fin último de promover proteger y apoyar a la lactancia materna, adentrándo- nos en la iniciativa del Baby Café y animando a que se realicen más estudios que avalen el verdadero impacto que éste tipo de iniciativas tienen sobre la sociedad; hemos desarrollado, mediante el estado de la cuestión y un diario de campo, un estudio cualitativo, que nos ha permitido explorar el fenómeno del Baby Café en el distrito 10 de Singapur, durante el mes de marzo de 2015.

Palabras clave: Baby Café, Apoyo, Iniciativa Internacional, Lactancia Materna.

\section{INTRODUCCIÓN}

El Baby Café es una iniciativa de apoyo a la lactancia materna, que coordina una red internacional de centros que brindan apoyo formal e informal a las madres y familias de los lactantes. Es un sistema novedoso ya que combina el apoyo formal proveniente de los profesionales sanitarios especializados en lactancia y el apoyo informal propio de los grupos o redes de apoyo a la lactancia (The Baby Café Charitable Trust, 2015).

El objetivo general de este trabajo consiste, en reflexionar sobre el fenómeno del Baby Café como recurso novedoso y conveniente dentro del mundo de la promoción, protección y apoyo a la lactancia materna a nivel internacional. Hablamos de fenómeno, ya que consideramos que la iniciativa del Baby Café se debe estudiar bajo el contexto de la fenomenología, entendiendo por ésta, como el estudio del fenómeno en sí mismo (Siles \& Solano, 2007) o dicho de otro modo, el estudio de las cosas tal y como se manifiestan y se muestran de manera empírica e intuitiva. Es por ello que hemos utilizado como principal instrumento en la investigación, el diario de campo desarrollado en el Baby Café de Singapur.

Para completar la reflexión sobre el fenó- 
meno del Baby Café, nos planteamos como objetivos específicos del estudio, en primer lugar:

- Explorar el concepto de la promoción, protección y apoyo a la lactancia materna, como objetivo internacional de salud; reflexionando sobre la efectividad de la combinación del apoyo formal e informal, como método efectivo para conseguir un apoyo eficaz, cercano y de calidad para las familias de los lactantes en todo el mundo.

- Profundizar en el fenómeno del Baby Café como iniciativa novedosa y modelo de apoyo formal e informal a la lactancia a nivel internacional, desarrollando su evolución desde su nacimiento, hasta nuestros días.

- Y por último, descubrir las percepciones, necesidades y experiencias de las usuarias del Baby Café de Singapur, por medio de un diario de campo.

Es por ello, que comienza nuestro estudio con el estado de la cuestión, en el que se abordan los cuatro primeros objetivos específicos planteados anteriormente; seguidamente desarrollaremos, en el apartado de material y método, el trabajo de campo desarrollado en el Baby Café de Singapur, completando de tal modo los cinco objetivos específicos del estudio.

\section{ESTADO DE LA CUESTIÓN}

Promoción, protección y apoyo a la lactancia materna como objetivo internacional de salud

La Organización Mundial de la Salud (OMS), afirma que la lactancia materna reduce la mortalidad infantil y tiene beneficios sanitarios que llegan hasta la edad adulta; estos beneficios no sólo son para el niño, también lo son para la madre, la comunidad y el medio ambiente en general. Para toda la población, se recomienda la lactancia materna exclusiva durante los seis primeros meses de vida y a partir de entonces, su refuerzo con alimentos complementarios, al menos hasta los dos años de edad del niño (OMS, 2015). Pero a pesar de conocer estos beneficios, encontramos que el abandono precoz y los bajos índices de lactancia materna suponen un problema común a nivel internacional (Baño Piñero, Canteras Jordana, Carrillo García, López Araez \& Martínez Roche, 2015). García Méndez (2010), afirma que la lactancia materna es uno de los fenómenos naturales más influenciados por la cultura (proceso bio-cultural). Tal vez por esta razón, podamos entender que existan grandes variaciones en los porcentajes de lactancia materna a lo largo de la historia y en distintas poblaciones dentro del mismo momento temporal.

Existe evidencia científica a cerca de los factores protectores y desfavorecedores de la lactancia materna, y aunque éstos difieran de un autor a otro, la mayoría coincide en que los profesionales sanitarios juegan un papel fundamental a la hora de apoyar, promocionar $y$ proteger la lactancia materna. Así mismo, establecer medidas de apoyo a la lactancia en los servicios en donde se trabaja con lactantes y técnicas de ayuda proporcionado por personal cualificado y consejeras de lactancia resulta de gran ayuda para mejorar los porcentajes de lactancia materna (Hernández Aguilar \& Aguayo Maldonado, 2005).

En el documento "Pruebas Científicas de los Diez Pasos hacia una Feliz Lactancia Natural", publicado en 1998 por la OMS, se identifican trece estudios controlados aleatorios o comparativos en los que se comprueba la efectividad del apoyo tras el alta sobre la lactancia materna. Crear un ambiente seguro y 
amigable, que brinde apoyo a las familias tras el post-parto es fundamental para proteger $y$ promover la lactancia materna. Sin embargo, no hay suficientes estudios que demuestren el potencial de los grupos de madres y de las promotoras comunitarias.

Puede que los grupos de apoyo informales sean más capaces que los servicios de salud formales de ofrecer la ayuda personal y frecuente que las madres necesitan para aumentar su confianza y superar las dificultades. Posiblemente, una combinación de apoyo día a día en la comunidad, respaldado por una atención más especializada en los servicios de salud cuando sea necesario, podría ser más eficaz que cualquiera de ellos por separado. (pag.84)

Se comprueba con esto que la idea de combinar el apoyo informal, como pueden ser los grupos de apoyo a la lactancia, con el apoyo formal proveniente de los profesionales sanitarios especializados en lactancia como matronas, consultores de lactancia, pediatras o médicos de familia, puede ser una actividad novedosa, eficaz y efectiva en términos de promoción y protección de la lactancia materna, tal y como resumimos en la gráfica I.
Tal vez, en base a lo anteriormente citado, la OMS, el Fondo Internacional de Emergencia de las Naciones Unidas para la Infancia (UNICEF) y la Iniciativa para la Humanización de la Asistencia al Nacimiento y la Lactancia (IHAN), publican dentro de los "Siete Pasos para una Feliz Lactancia Natural”, en su Manual para la acreditación de Centros de Salud IHAN (2014):

- Paso 2: Capacitar a los trabajadores para que puedan poner en práctica la normativa de lactancia.

- Paso 6: Proporcionar una atmósfera de acogida a las madres y lactantes.

- Paso 7: Disponer de un taller de lactancia y fomentar la formación y el contacto con grupos y recursos de apoyo a la lactancia en la comunidad.

Como requisitos imprescindibles para obtener dicho galardón. Comprobando una vez más la importancia de combinar el apoyo formal e informal).

El apoyo formal, entendiendo por éste, como cualquier profesional sanitario capaz de detectar, derivar o tratar adecuadamente, cualquier problema relacionado con la lactancia, proporciona una fuente de información

\section{Gráfico I}

Beneficios de la combinación del apoyo formal e informal.

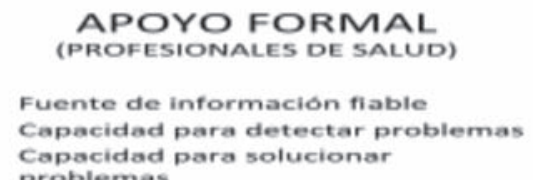

APOYO FORMAL

(PROFESIONALES DE SALUD)

Capacidad para solucionar problemas
APOYO INFORMAL

(GRUPOS DE APOYO)

Accesibilidad

Posibilidad de involucrar a la familia

Ambiente seguro, amigable, distendido, flexible y cercano.

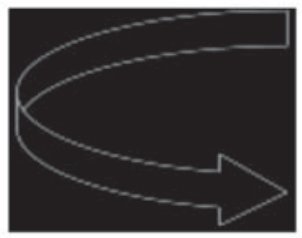

APOYO EFICAZ

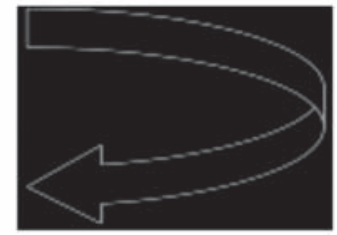

Fuente: Gráfico de elaboración propia 
fiable a las familias de niños lactantes, necesaria para superar las dificultades que puedas aparecer durante esa etapa. En cambio, encontramos en muchas ocasiones, que a los profesionales de salud, les es difícil brindar un seguimiento adecuado, proporcionando servicios no siempre accesibles o cercanos; es por esto que las madres pueden ser reacias a buscar ayuda en el sistema formal de salud cuando aparecen problemas o dificultades con la lactancia (OMS, 1998). Las redes de apoyo informales, como los grupos y recursos de apoyo a la lactancia en la comunidad, aportan accesibilidad, un ambiente distendido, seguro, flexible y amigable, que hacen que las madres y familias de lactantes se sientan más cómodas a la hora de consultar sus problemas sobre lactancia; también permiten involucrar a la familia inmediata de la madre, como los compañeros, abuelas o amigas, que tanta influencia tienen sobre la decisión de lactar (Álvarez, 2013; López, Molina, Martínez, Frutos y Molina, 2012). Numerosos estudios (Hoddinott \& Pill,1999; Lavender, McFadden \& Baker, 2006; McFadden \& Tooler, 2006; Scott \& Mostyn, 2003) concluyen que las experiencias y percepciones derivadas del apoyo informal, tales como grupos de apoyo a la lactancia o amigos y familiares, tienen una fuerte influencia en la intención de lactar y en la permanencia de ésta (NCT, 2014).

Además de los factores protectores de la lactancia materna que acabamos de comentar, no podemos pasar por alto la importancia de la empatía dentro del apoyo formal e informal como requisito imprescindible para la consejería en lactancia materna (OMS y UNICEF, 1993; Simões, Soares, Duarte, Bracarense y Cortes, 2015). Nogales (2012) define la empatía como "la vivencia de la experiencias de sujetos ajenos, la vivencia de experiencias de otros. Vivencia de experiencias de otros que abren en uno, horizontes distintos al que es propiamente personal", y pone de manifiesto en su estudio, la necesidad e importancia de su aplicación para la enfermería en el contexto familiar.

Parece que en base a lo anteriormente expuesto, queda claro que la promoción, protección y apoyo a la lactancia materna se compone de varios factores que en conjunto resultan mucho más efectivos que por separado. La iniciativa del Baby Café es un claro ejemplo de combinación de apoyo a la lactancia formal e informal; pero para poder entender éste fenómeno, es necesario conocer más a fondo el concepto del Baby Café, por esta razón pasamos a desarrollamos a continuación su concepto, objetivos y evolución:

\section{El Baby Café}

El Baby Café es un servicio internacional de apoyo a la lactancia que combina los beneficios de un ambiente distendido e informal propio de un grupo de apoyo a la lactancia, pero a la vez, liderado por profesionales en lactancia, capaces de ayudar de manera profesional, accesible y eficaz a las madres que lo necesiten.

Tal y como aparece en el Informe Anual del Baby Café 2005-2006 (The Baby Café Charitable Trust, 2006), los objetivos generales que pretende la iniciativa son:

1) Promover la salud física y psicológica de las madres y los niños a través de la capacitación y educación en las técnicas sobre lactancia materna.

2) Fomentar la educación acerca de los beneficios para la salud de la lactancia materna tanto inmediatos como a largo plazo en la sociedad en general y sobre todo en aquellas personas interesadas en el cuidado de los niños. 
3) Mejorar la formación del personal voluntario que trabaja en esta área mediante la provisión de capacitación pertinente.

Y a nivel específico (Baby Café Local, 2013):

- Proveer un modelo de apoyo social a la lactancia en un ambiente cómodo y cercano.

- Proporcionar un servicio universal, accesible a todos los niveles de la sociedad, en especial a los grupos más desfavorecidos o marginales.

- Procurar un servicio profesional capaz de ofrecer información fiable y dar apoyo a la lactancia, de manera accesible y cercana. Siendo capaz de detectar problemas relacionados con la lactancia y tratarlos o derivarlos cuando sea necesario.

- Respetar y mantener el estándar de calidad propuestos por la organización.

- Ayudar a que más mujeres lacten, contribuyendo a que superen sus problemas de lactancia de manera oportuna y eficaz. Favoreciendo la lactancia materna para que ésta sea una experiencia positiva para todas ellas y apoyándoles para que sean capaces de lactar hasta donde se lo propongan.

En líneas generales, se persigue que las mujeres tengan una experiencia positiva con su lactancia, fomentando la cultura de la lactancia materna, y aumentando con ello la incidencia y permanencia de ésta.

\section{Nacimiento y evolución del Baby Café}

Por los motivos anteriormente mencionados y con la intención de implantar como parte del plan de UNICEF para la acreditación de la IHAN, se crea en 2000 por primera vez el concepto del Baby Café por dos consultoras internacionales de lactancia, Catherine Pardoe y Juli Williams (The Baby Café Charitable Trust, 2015). El primer Baby Café se fundó en West Sussex (Inglaterra), como parte del Departamento de Iniciativa de Alimentación Infantil Saludable del Sistema Nacional Inglés de Salud (NHS Trust). Pronto, éste modelo fue creciendo en popularidad llegando a incluirse dentro del Protocolo de Buenas Prácticas e Innovación en Lactancia Materna del NHS (Department of Health, 2004).

En diferentes partes de Inglaterra se empezó a solicitar la creación de dicho modelo como parte de los servicios de promoción a la lactancia materna del Sistema Nacional de Salud. Fue entonces cuando se desarrolló una guía donde se especificaba paso a paso cómo solicitar, crear y evaluar un centro Baby Café (Baby Café, 2012).

Gracias a la gran aceptación que esta iniciativa obtuvo, se decidió internacionalizar el concepto del Baby Café, registrando la marca y el logo de Baby Café. De este modo, cualquier centro que quisiese acreditarse como tal, tendría que pagar una cuota anual que le permitiese acceder a la estructura nacional de apoyo, así como asegurar el asesoramiento y la supervisión del centro garantizando cumplir con los requisitos de calidad requeridos por la organización. Las 12 normas de calidad exigidas por la organización son (Baby Café Local, 2013):

1. Respetar la marca “The Baby Café”. Los centros acreditados deberán usar el mismo nombre y logo registrado por la organización.

2. Personal cualificado. La persona responsable de solicitar la acreditación deberá poseer como mínimo una titulación sanitaria equivalente al Grupo 6 (visitador sanitario, enfermera, matrona o consultor internacional de lactancia; todos ellos con una experiencia mínima requerida).

3. Trabajo multidisciplinar. Los centros Baby Café deberán involucrar y contar con 
la colaboración de distintos profesionales de salud así como con voluntarios y grupos de apoyo a la lactancia.

4. Ambiente acogedor. Los centros acreditados proporcionarán un ambiente seguro, cálido y hospitalario en el que se ofrecerá bebida y comida para atraer a las mujeres regularmente (apoyo informal). Igualmente se asegurará espacio y tiempo suficiente para atender madres con problemas en lactancia (apoyo formal).

5. Modelo de apoyo social. El modelo de Baby Café asegurará un apoyo formal, por parte de los profesionales de salud o consultores de lactancia, que permitirá identificar madres y niños con necesidades especiales tanto físicas como mentales, respondiendo correctamente a sus requerimientos bio-psico-sociales. Así mismo se asegurará un trato amable y cercano a aquellas que tengan problemas sociales o emocionales.

6. Promover y apoyar la lactancia. El Baby Café acogerá a mujeres en periodo prenatal y postnatal, animando a que éstas continúen lactando exclusivamente más de 6-8 semanas, usando el modelo de refuerzo positivo. La mayoría de casos de destete no son planificados, el $80 \%$ de las mujeres que dejan de lactar durante las primeras seis semanas de vida del bebé lo hicieron antes de lo planeado y más de tres cuartas partes de las madres hubieran preferido seguir lactando durante más tiempo (NCT, 2014).

7. Atención a toda la comunidad. Cualquier mujer, provenga del sector de la comunidad que provenga, deberá de sentirse motivada para acceder al centro, recibiendo siempre información y apoyo apropiado.

8. Servicio accesible. El acceso al centro debe de garantizar unos mínimos. Se deben de cumplir al menos tres de los seis requisitos que se plantean a continuación: un lugar para aparcar los carritos, acceso al centro por medio de autobús, cerca de otros servicios de salud o tiendas, buena ubicación en la que haya acceso al un parking asequible por las usuarias, capacidad por parte de los trabajadores para hablar varios idiomas o instrumentos que faciliten la comunicación con mujeres que tengan limitaciones con el idioma.

9. Capacidad para derivar correctamente. Los profesionales que trabajen en el Baby Café deberán ser capaces de identificar correctamente los problemas de lactancia y ayudar a solventarlos o derivarlos a otro especialista si lo requieren.

10. Fuente de información de alta calidad. Los centros contarán con información sobre lactancia fiable y de calidad, de fácil comprensión y accesible para toda la familia. Tales como posters, folletos, o soportes audiovisuales. Se proporcionará también información sobre otros servicios de apoyo a la lactancia.

11. Revisiones periódicas y de mejora. La organización del Baby Café se reúne periódicamente para hacer revisiones sobre sus servicios, reflexionar sobre el trabajo realizado, abordar propuestas de mejora y planes de actuación.

12. Proporcionar datos fiables. Cada 31 de Enero se recogen y analizan todos los datos solicitados para cada centro y éstos se estudian junto con el Sistema Inglés de Estadística. Así mismo, se publica un trabajo anual en el que se exponen y analizan dichos datos.

En 2005 la iniciativa del Baby Café se convierte en "The Baby Café Charitable Trust”, consiguiendo así una estructura más estable y sólida. Un año más tarde se publica el Manual del Baby Café, en el que se proporciona información detallada sobre cómo desarrollar un Centro Baby Café con éxito y se pone de ma- 
nifiesto la importancia de controlar el cumplimiento de las doce normas anteriormente mencionadas y asegurar la calidad mínima exigida para cada centro. El objetivo principal de este control es asegurar un apoyo a la lactancia continuo, accesible y de calidad para toda la sociedad, garantizando así el número creciente de centros y madres que acuden a éstos (The Baby Café Charitable Trust, 2015). Finalmente en 2010 "The Baby Café Charitable Trust" se fusiona con el NCT (National Childbirth Trust), la mayor organización inglesa sin ánimo de lucro que da apoyo a las familias en el periodo prenatal y postnatal (NCT, 2014), permaneciendo esta unión hasta día de hoy.

El desarrollo del concepto del Baby Café, como iniciativa novedosa de apoyo formal e informal a la lactancia materna, ha tenido un crecimiento exponencial como se puede comprobar en la Tabla I. El primer centro se abrió en el 2000, y en 2013 ya habían un total de 155 centros acreditados en todo el mundo; pero esta cifra tan solo representa los centros que mantienen la acreditación a esa fecha, en cambio, si contamos los 165 centros que no han podido mantener la acreditación o los que han cerrado a lo largo de esos años, nos encontraríamos con una cifra de 320 Baby Cafés abiertos entre el 2000 y 2013 a nivel internacional. Inicialmente el crecimiento de esta iniciativa fue principalmente por Reino Unido, pero rápidamente empezó a desarrollarse por todo el mundo. A 31 de diciembre de 2013 habían 108 Baby Cafés en Inglaterra, 41 en Estados Unidos, 4 en México, 1 en Nueva Zelanda y 1 en Singapur (Baby Café Local, 2013). La influencia de los Baby Cafés es significativa, o por lo menos abarca a un número importante de mujeres. Se calcula que solo en los Baby Cafés de Inglaterra durante el año 2013 pasaron más de diez mil mujeres de las cuales, más de una cuarta parte acudieron al centro seis veces o más (Baby Café Local, 2013), si esto lo aplicamos al resto de centros y a lo largo de los años que ésta iniciativa lleva funcionando, las cifras serían considerables.

\section{MATERIAL Y MÉTODO}

Para dar respuesta a los objetivos planteados en el estudio, se ha abordado una meto-

Tabla I

Evolución del número total de Baby Cafés de 2001 a 2013.

(Baby Café Local, 2013; The Baby Café Charitable Trust, 2006)

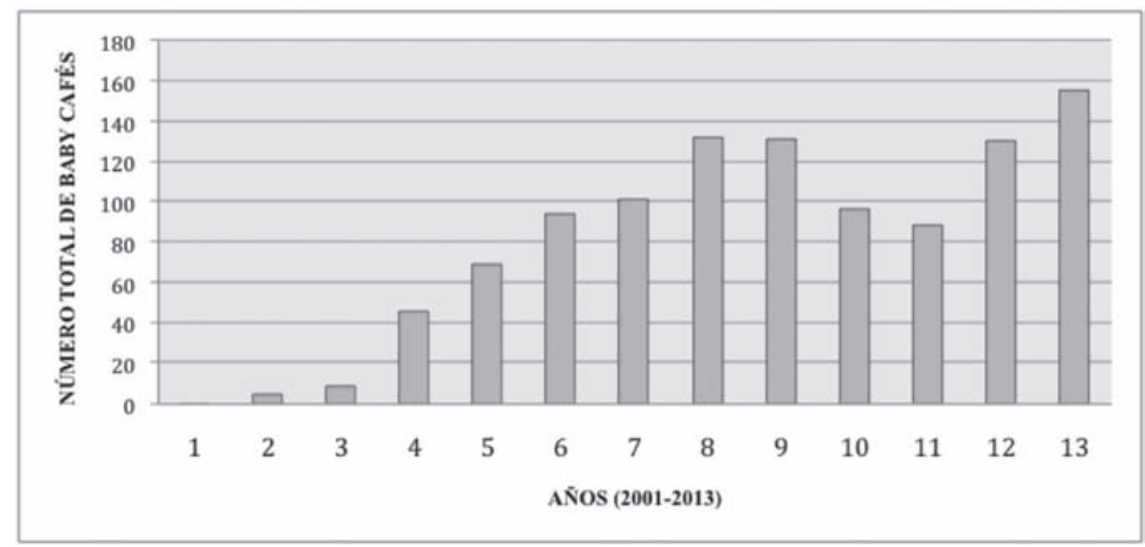

Tabla de elaboración propia a partir de los documentos anteriormente mencionados 
dología cualitativa, que como la define Taylor y Bogdan (1987), se trata de una investigación que produce datos descriptivos: las propias palabras de las personas, habladas o escritas y la conducta observable, permiten profundizar en la teoría fenomenológica de manera intuitiva y en base a la experiencia; permitiendo reflexionar sobre las acciones realizadas en una determinada situación. El Baby Café de Singapur ha sido el principal fenómeno a explorar en este estudio, siendo el diario de campo el instrumento utilizado para poder llevarlo a cabo.

El estudio se ha desarrollado en el distrito 10 de Singapur o distrito Central, más concretamente, en el único centro acreditado como Baby Café de Singapur. Las participantes del estudio han sido las usuarias del Baby Café de Singapur, que han acudido al centro con sus bebés durante el mes de marzo de 2015 y que habiendo sido previamente informadas del proyecto han dado su consentimiento para poder realizarlo. La investigación se ha elaborado bajo el firme compromiso de respetar tanto las normas éticas que rigen los estudios científicos, como el anonimato de los participantes de este estudio, siguiendo la Ley Orgánica de Protección de Datos de Carácter Personal.

Dentro de las limitaciones del estudio hay que tener en cuenta que al haber informado a las usuarias sobre el proyecto de estudio y habiendo pedido previamente su consentimiento para éste, puede que la técnica de observación haya ejercido una presión que haya disminuido o bloqueado la espontaneidad en algunas participantes, pero como afirman Taylor y Bogdan (1987), la investigación cualitativa es sensible a los posibles efectos debido a la presencia del investigador, pero a su vez es necesaria para comprender a las personas dentro de su propio marco de referencia. Es por ello, que teniendo en cuenta la naturaleza de la investigación cualitativa, consideramos adecuada la técnica.

\section{Experiencia en el Baby Café de Singapur: diario de campo}

El diario de campo se ha desarrollado en cuatro sesiones de observación, de las cuales, tres han sido observaciones relacionadas con el contexto y la población a estudiar y una última, en las que hemos intervenido como profesionales.

\section{DESCRIPCIÓN}

Observación 1: Contexto de ocurrencia (2 de marzo de 2015).

Objetivo: Describir el contexto en el que se desarrolla el Baby Café de Singapur.

Anotaciones: Dentro del un centro comercial, en la tercera planta, hay un centro de atención prenatal y postnatal en el que trabajan diferentes profesionales relacionadas con el embarazo y crianza. Así podemos encontrar matronas, enfermeras, visitadoras sanitarias (health visitor), consultoras internacionales de lactancia o instructoras de yoga y pilates pre y post natal. Algunas de las actividades que se ofertan en el centro son, educación prenatal, clases de primeros auxilios, introducción a los sólidos, incorporación laboral y lactancia materna, consulta del niño sano, consulta de lactancia, consulta de la matrona, visita domiciliaria, yoga, pilates, masaje del bebé y el Baby Café entre otras. El Baby Café se organiza semanalmente, los lunes de 2 a 4 de la tarde. Se invita a todas las mamás o futuras mamás, así como a los familiares, que acuden al centro por otros motivos y también se anuncia en la página web del centro. La sala donde se desarrolla la actividad es bastante 
amplia y luminosa, con cómodos sillones con cojines a todo alrededor. También hay dos soportes cambiadores para los bebés. Hay una mesa en un lateral en donde hay por lo general galletas o pasteles con dispensadores de café, tee y agua. En el suelo hay mantitas de colorines para los bebés, el suelo está todo enmoquetado, y solo se puede acceder sin calzado a la sala. En las paredes hay imágenes de bebés y madres dando el pecho, así como carteles que hacen alusión a los beneficios, la técnica o los principales problemas y soluciones sobre lactancia. Se puede encontrar a la entrada numerosos contactos de otros recursos de apoyo a la lactancia y diferentes profesionales a los que pueden acudir las usuarias del centro. En la recepción del centro hay productos relacionados con la lactancia y crianza que se pueden comprar, como pañuelos porta bebés, sacaleches, cojines de lactancia, etc. Además de la sala en donde se desarrolla el Baby Café hay otra sala anexa más pequeña, en la que se llevan a cabo otras actividades como clases o consultas al mismo tiempo que transcurre el Baby Café.

Observación 2: Relaciones y situaciones de los sujetos (9 de marzo de 2015).

Objetivo: Acercarse a la población a estudiar: las usuarias del Baby Café de Singapur.

Anotaciones: La mayoría de mujeres que acuden con sus bebés al Baby Café suelen ser madres que están dando el pecho o que lo combinan con leche de fórmula, también acuden, aunque en menor número, madres que no dan el pecho, embarazadas o familiares de éstas. La edad de los bebés también varía mucho, pero por lo general suelen venir bebés de entre 1 semana y 6 meses, aunque también se pueden encontrar niños mayores de un año o hermanos de éstos. El número de usuarias que acuden al Baby café suele ser numeroso, aun- que en periodo vacacional se suele notar un descenso importante de mujeres, por lo general los grupos suelen oscilar de 10 a 20 mujeres y sus respectivos bebés. La actividad del Baby Café está liderada por dos profesionales que trabajan en el centro, que suelen ser matronas y/o consultoras internacionales de lactancia. En la recepción hay dos trabajadoras más que hacen labores administrativas y que atienden a las usuarias que quieran comprar algún producto o apuntarse a cualquier otra actividad que oferta el centro. El perfil de las usuarias suele coincidir con mujeres expatriadas, de diferentes nacionalidades, sobre todo europeas y chinas, siendo la comunidad más numerosa, la inglesa. Las mujeres locales también acuden a esta actividad aunque en menor proporción. El idioma que se habla es el inglés, pero en algunas ocasiones acuden mujeres con limitaciones en el idioma, con las que se trabaja más intensivamente para salvar dicha limitación. Los profesionales que trabajan en el Baby Café suelen hablar varios idiomas.

\section{Imagen I}

\section{Imágenes del Baby Café de Singapur}

(Marzo, 2015).

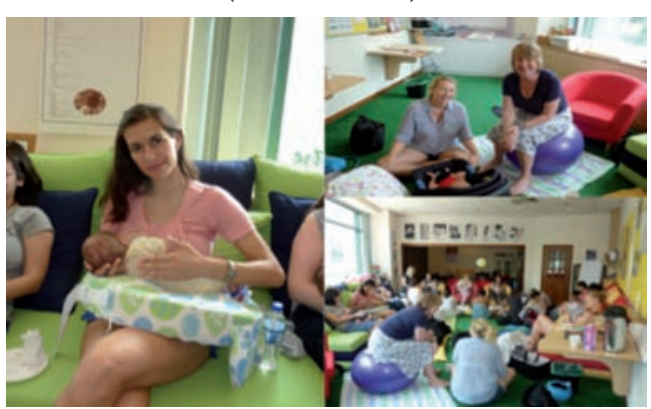

Fuente: Mother and Child, Singapur, Marzo del 2015

Observación 3: Correlaciones y situaciones.

Objetivo: Observar cuidadosamente qué problemas o consultas se dan en el Baby Café (16 de marzo de 2015).

Anotaciones: Las madres que acuden al 
centro, inicialmente acuden para consultar su problema de lactancia con algún profesional destinado a tal efecto, pero después de probar por primera vez la actividad, suelen repetir con mucha frecuencia ya que terminan haciendo amistad con otras madres, puesto que se trata de una reunión informal y distendida, es por ello que además de servirles de apoyo, también le sirve de entretenimiento y actividad social con niños. Las usuarias van acercándose a las dos trabajadoras que lideran la actividad y le van preguntando sus dudas o consultas sobre lactancia y crianza de manera informal, en ocasiones, entre las propias usuarias resuelven sus dudas, sirviéndose de la experiencia de otras madres que han pasado por la mima situación que ellas. Las madres se suelen encontrar relajadas y cómodas para consultar cualquier duda. Las consultas más frecuentes son problemas de agarre o enganche del bebé al pecho, cansancio o problemas con el sueño del bebé, grietas en el pezón, cómo y cuándo incorporar los sólidos, recomendaciones antes de incorporarse a trabajar y rechazo del pecho por parte del bebé. Casi todas las madres van buscando en esta actividad un apoyo social a su situación, ya que en muchas ocasiones se sienten solas o incomprendidas.

Observación 4: Observación y participación.

Objetivo: Participar como matrona en el Baby Café (23 de marzo de 2015).

Anotaciones: El trabajo con madres y bebés, es un trabajo muy gratificante pero a la vez muy dedicado, las usuarias del Baby Café suelen ser madres que buscan un apoyo que no encuentran en la sociedad, lo que conlleva a que en muchas ocasiones se sientan solas o incomprendidas como hemos mencionado anteriormente; es por ello que la empatía y la comunicación cercana y accesible son requi- sitos imprescindibles para trabajar con estas mujeres. Hay consultas que son bastante sencillas de resolver, pero en otras ocasiones nos vemos obligados a derivar a las usuarias a otros especialistas que colaboran con el centro como pediatras, médicos de familia, especialistas en frenillos, quiroprácticos o ginecólogos. El ambiente que se respira en el Baby Café es amable, relajado y seguro. Las consultas se resuelven sobre la marcha mientras conversamos de manera informal.

Testimonio de una de las usuarias del Baby Café de Singapur:

"I've been attending the Baby Café at Mother \& Child from my baby's second week of age. At that time motherhood was totally new to me and it felt great to be able to meet with other mums in similar situation and to get great support and advice from the available midwifes there. The Baby Café was recommended to me by a friend towards the end of my pregnancy but I didn't give it much thought at the time. However, a few days after my baby was born I had many questions and felt quite isolated, so I managed to get over any initial shyness and fear of going out with a newborn, and there has been no looking back.

I try to attend the Baby Cafe every week. Not only it gives me a great opportunity to spend time with other mums, exchange ideas, and get my baby to meet other babies and spend some time in a stimulating environment. I also get all the time I need with a midwife, usually my Spanish compatriot Isabel, to ask any questions and clear up any doubts about feeding, baby behaviour, sleeping issues and generally getting practical tips to make each day of my new motherhood chapter that little bit easier.

We had some serious issues in our early breastfeeding days, and attending the Baby Cafe weekly, allowed me to correct latching proble- 
ms, have great support and hear from other mums who had succeeded in breastfeeding their babies. It is not unusual for me to come to the Baby Cafe with a list of questions, as I'm confident that I will get great answers and help.

I would strongly recommend to any new (and not-so-new) mums to join us every Monday. The atmosphere is positive and friendly, the setting is comfortable with lots of comfy cushions for mummy and baby, and there is always a yummy cake or delicious homemade cookies! Come join us at the Baby Cafe!"

"He estado asistiendo al Baby Café en Mother and Child desde que mi bebé tenía dos semanas. En aquel momento la maternidad era totalmente nueva para mí y me sentó genial poder reunirme con otras madres en la misma situación que yo y conseguir un gran apoyo $y$ asesoramiento por parte de las matronas disponibles allí. Una amiga me recomendó el Baby Café hacia el final de mi embarazo, pero no le di mucha importancia en aquél momento. Sin embargo, pocos días después del nacimiento de mi bebé tenía muchas preguntas y me sentí bastante aislada, así que me las arreglé para salvar la timidez inicial y el miedo a salir con un recién nacido, y lo hice, no había vuelta atrás.

Intento ir al Baby Café cada semana. No sólo es una gran oportunidad para pasar tiempo con otras madres, intercambiar ideas, y hacer que mi bebé conozca otros niños y pasar algún tiempo en un ambiente estimulante. También tengo todo el tiempo que necesito con la matrona, por lo general mi compatriota española Isabel, de hacer preguntas y aclarar cualquier duda acerca de la alimentación, el comportamiento del bebé, problemas para dormir y en general conseguir consejos prácticos para hacer que cada día de mi nuevo capítulo de la maternidad sea un poco más fácil.
Hemos tenido algunos problemas serios en nuestros principios con la lactancia materna y asistir a la Baby Café semanalmente, me permitió corregir problemas de enganche, tener un gran apoyo y escuchar a otras madres que habian logrado amamantar a sus bebés. No es raro en mí ir al Baby Café con una lista de preguntas, ya que estoy segura de que voy a obtener grandes respuestas y ayuda.

Recomiendo encarecidamente a todas las nuevas (y no tan nuevas) madres a unirse a nosotros todos los lunes. El ambiente es positivo $y$ amable, el lugar es confortable, con un montón de cojines cómodos para mamás y bebés, y siempre hay deliciosas tartas o galletas caseras! Ven y únete a nosotros en el Baby Café! "

Traducción personal.

\section{DISCUSIÓN}

Como hemos comentado en apartado de introducción, parece que queda claro que la combinación del apoyo formal e informal es muy efectivo en términos de promoción y protección de la lactancia materna. Un ambiente cercano, accesible, relajado y empático, propio del apoyo informal procedente de las redes o grupos de apoyo a la lactancia, unido a un apoyo formal, como son los profesionales sanitarios especializados en lactancia, es la clave para acceder e interferir de manera positiva en la duración y permanencia de la lactancia materna.

Nuestra experiencia como profesional especializado en lactancia en el Baby Café de Singapur, coincide con lo que la evidencia científica avala en cuanto a la efectividad de éste tipo de servicios para la comunidad. Por lo general, las usuarias del Baby Café hablan muy bien de la iniciativa y la recomiendan muy a menudo; tal vez sea por eso que la mayoría de mujeres que llegan por primera 
vez al centro, sea por medio de la recomendación de algún amigo, familiar o profesional (Baby Café Local, 2013).

Por todos los motivos anteriormente expuestos, recomendamos que iniciativas como la del Baby Café, se impulsen y promuevan con más fuerza a nivel internacional, bien por medio de su difusión y desarrollo a través de organismos públicos o privados de salud, o bien por el fomento de la investigación de éste tipo de fenómenos.

\section{CONCLUSIONES}

A través de éste estudio, hemos profundizado en el concepto del Baby Café, llegando a la conclusión de que éste tipo de iniciativas son muy beneficiosas para la sociedad en la que vivimos, en la que los bajos índices de lactancia suponen un problema de salud mundial. Como hemos comprobado en el estado de la cuestión, la lactancia materna es beneficiosa no sólo para la salud del lactante, también lo es para las familias, el medo ambiente y la sociedad en general; es por ello que cada vez se hacen más necesarias, medidas de promoción protección y apoyo a la lactancia materna que contribuyan a solucionar dicho problema.

La evolución del Baby Café ha sido exponencial, su crecimiento e internacionalización se deben, en gran parte, al éxito de éste tipo de modelos de apoyo a la lactancia materna. No suponen un gran coste económico para la sociedad y en cambio mejoran la calidad del sistema de salud, enriquece la percepción que las mujeres tiene sobre sus lactancias y contribuyendo a mejorar la permanencia e incidencia de la lactancia materna en la comunidad.

Mediante el diario de campo hemos tenido la oportunidad de aproximarnos al fenómeno del Baby Café de Singapur, viendo más de cerca cómo se desarrolla el día a día de éste servicio. El ambiente que se respira es familiar y cercano, las usuarias suelen volver en numerosas ocasiones, lo que nos sugiere que es una iniciativa que funciona y que cumple su principal objetivo, que es ayudar y apoyar a las madres durante sus lactancias. El testimonio de una de las usuarias ratifica todo lo anteriormente expuesto y pone de manifiesto el alto grado de satisfacción percibida por ésta al contar con los servicios del Baby Café de Singapur.

Con este estudio queda clara la importancia y necesidad que los profesionales sanitarios especializados en lactancia, en combinación con el servicio del Baby Café, juegan para la sociedad; aun así, queda mucho por hacer en cuanto a investigación sobre éste tipo de iniciativas de apoyo a la lactancia; es por ello que animamos a que se realicen más estudios que avalen el verdadero impacto que el Baby Café tiene sobre la sociedad, con el fin último de promover proteger y apoyar a la lactancia materna de forma unánime.

\section{BIBLIOGRAFÍA}

- Álvarez Zendejas, P. M. (2013). Proyecto Terminal Profesional: "Propuesta de intervención para promover una práctica correcta de la lactancia materna exclusiva en adolescentes embarazadas de Santo Tomás Ajusco”. (Maestría en Salud Pública Área de Concentración: Epidemiología para obteneción del grado de maestría en Salud Pública). Instituto Nacional de Salud Pública. Escuela de Salud Pública de México. Recuperado el 15 de enero de 2015 de http://www.inspvirtual.mx/CentroDocumentacion/cwisBancoPF/SPT--DownloadFile. php? Id $=560$

- Baby Café. (2012). Supplementary Information Sheets. Baby Café National Co-ordinators. September 2012. Recuperado el 24 de marzo de 2015 de http://www.google. com.sg/url? sa $=\mathrm{t} \& \mathrm{rct}=\mathrm{j} \& \mathrm{q}=\& \mathrm{esrc}=\mathrm{s} \&$ source $=\mathrm{web} \& \mathrm{~cd}=$ 2\&ved=0CCEQFjAB\&url=http\%3A\%2F\%2Fthebabyca 
fe.org\%2Findex.php\%3Foption\%3Dcom_rubberdoc\% 26view\%3Ddoc\%26id\%3D153\%26format\%3Draw\&ei= NsAQVdXwIpe3uQS8gYHIDg\&usg=AFQjCNHWEiei eNYQulj35Ee0mfEtlHyM5Q\&bvm=bv.89184060,d.c2E

- Baby Café Local. (2013). Report on Baby Café Services during 2013. Recuperado el 24 de marzo de 2015 de http://www.nct.org.uk/sites/default/files/related_documents/Baby\%20Cafe\%202013\%20Annual\%20Report\%202014\%20External\%20use_0.pdf

- Baño Piñero, I., Canteras Jordana, M., Carrillo García, C., López Araez, A. \& Martínez Roche, M. E. (2015). Desarrollo y validación de un instrumento diseñado para medir el impacto de las redes de apoyo a la lactancia materna. Nutr Hosp. 31(4), 1525-32

- Department of Health. (2004). Good practice and innovation in breastfeeding. Recuperado el 19 de enero de 2015 de http://webarchive.nationalarchives.gov.uk/+/www. dh.gov.uk/en/Publicationsandstatistics/Publications/ PublicationsPolicyAndGuidance/DH_4097202

- García Méndez, J. A. (2010). Eficacia diferencial de los programas de apoyo a madres con intención de lactar sobre la exclusividad y duración de la lactancia materna: Un estudio meta-analítico. (Tesis Doctoral). Universidad de Murcia. Murcia.

- Hernández Aguilar, M. T. \& Aguayo Maldonado, J. (2005). La lactancia materna: Cómo promover y apoyar la lactancia materna en la práctica pediátrica. Recomendaciones del Comité de Lactancia de la AEP. An Pediatr. 63(4), 340-56.

- Hoddinott, P. \& Pill, R. (1999). Qualitative study of decisions about infant feeding among women in the east end of London. BMJ. 318, 30-4.

- Lavender, T., Mc Fadden, C. \& Baker, L. (2006). Breastfeeding and family life. Matern.Child Nutr. 2(3), 145-55.

- López Marín, R., Molina Rodríguez, A., Martínez Roche, M., Frutos Molina, D. \& Molina Rodríguez, T. (2012). Figura del padre en relación a la crianza: pasado, presente y futuro. Cultura De Los Cuidados. 16(32), 12-7. doi:http://dx.doi.org/10.7184/cuid.2012.32.02

- Mc Fadden, A. \& Toole, G. (2006). Exploring women's views of breastfeeding: a focus group study within an area with high levels of socio- economic deprivation.
Matern.Child Nutr.2(3), 156-68.

- NCT. (2014). Baby Feeding Policy 2014. DRAFT for discussion. Recuperado el 24 de marzo de 2015 de http://www.nct.org.uk/sites/default/files/related_documents/2014\%20Baby $\% 20$ Feeding $\% 20$ policy $\% 20$ $\% 282 \% 29 . p d f$

- Nogales Espert, A. (2012). La empatía según Edith Stein y sus aplicaciones en enfermería en el contexto familiar. Cultura De Los Cuidados. 12(24), 119-33. Recuperado de http://culturacuidados.ua.es/enfermeria/article/ view/302/603

- Organización Mundial de la Salud (OMS). (2015). Lactancia materna. Salud de la madre, el recién nacido, del niño y del adolescente. Recuperado el 16 de febrero de 2015, de http://www.who.int/maternal_child_adolescent/topics/newborn/nutrition/breastfeeding/es/\#

- OMS. (1998). Prueba Científica de los Diez Pasos Hacia una Feliz Lactancia Natural. División de Salud y Desarrollo del Niño. Ginebra. Recuperado el 9 de noviembre de 2014 de http://whqlibdoc.who.int/hq/1998/WHO_ CHD_98.9_spa.pdf?ua=1

- OMS \& UNICEF. (1993). Curso de Capacitación Manual del Participante. Consejería en Lactancia Materna. Recuperado el 3 de marzo de 2015 de http://www.unicef.org/ argentina/spanish/Consejeria_en_Lactancia_Materna. pdf

- OMS, UNICEF \& IHAN. (2014). Estrategia Centros de Salud IHAN. Revisada, actualizada y ampliada para la atención integral en los centros de salud. Manual para la acreditación IHAN-España. 2014. Recuperado el 19 de febrero de 2015 de http://www.ihan.es/docs/documentacion-acreditacion/centros_salud/Manual_para_la_ acreditacion_IHAN_en_AP.pdf

- Scott, J. A. \& Mostyn, T. (2003). Women's experiences of breastfeeding in a bottle-feeding culture. J Hum Lact. 19(3), 270-7.

- Siles González, J., Solano Ruiz, M. \& Cibanal, J. (2012). El origen fenomenológico del "cuidado" y la importancia del concepto de tiempo en la historia de la enfermería. Cultura De Los Cuidados, 12(21), 19-27. Recuperado de http://culturacuidados.ua.es/enfermeria/article/ view/270/539 


\section{Cultura de las Cuidados}

- Simões, A., Soares, H., Duarte, J., Bracarense, C. \& Cortes, R. (2015). La humanización en el proceso de formación académica de los profesionales de la salud. Cultura De Los Cuidados. 18(40), 72-81. doi:http://dx.doi. org/10.7184/cuid.2014.40.10

- Taylor, S. J. Bogdan, R. (1987). Introducción a los métodos cualitativos de investigación. La búsqueda de significados. Ediciones Paidós Ibérica. S. A. Barcelona.

- The Baby Café Charitable Trust. (2006). ANNUAL REPORT and ACCOUNTS 2005 2006. Including information from the National Statistics and Evaluation 2005.
Recuperado el 23 de marzo de 2015 de http://www.google.com.sg/url? sa $=t \& r c t=j \& q=\& e s r c=s \&$ source $=$ web \& $\mathrm{cd}=2 \& v e d=0 \mathrm{CCEQFjAB} \& u r l=h t t p \% 3 \mathrm{~A} \% 2 \mathrm{~F} \% 2 \mathrm{Fwww}$. thebabycafe.org\%2Findex.php\%3Foption\%3Dcom_rub berdoc\%26view\%3Ddoc\%26id\%3D16\%26format\%3Dr aw\&ei=_b0QVdPkAo_buQTzwoHQBg\&usg=AFQjCN Hk1bR37mtpsgRMfdAA9MPElycIJw\&bvm=bv. 891840 60, d.c2E

- The Baby Café Charitable Trust. (2015). The Baby Café. Recuperado el 19 de enero de 2015 de http://www.thebabycafe.org

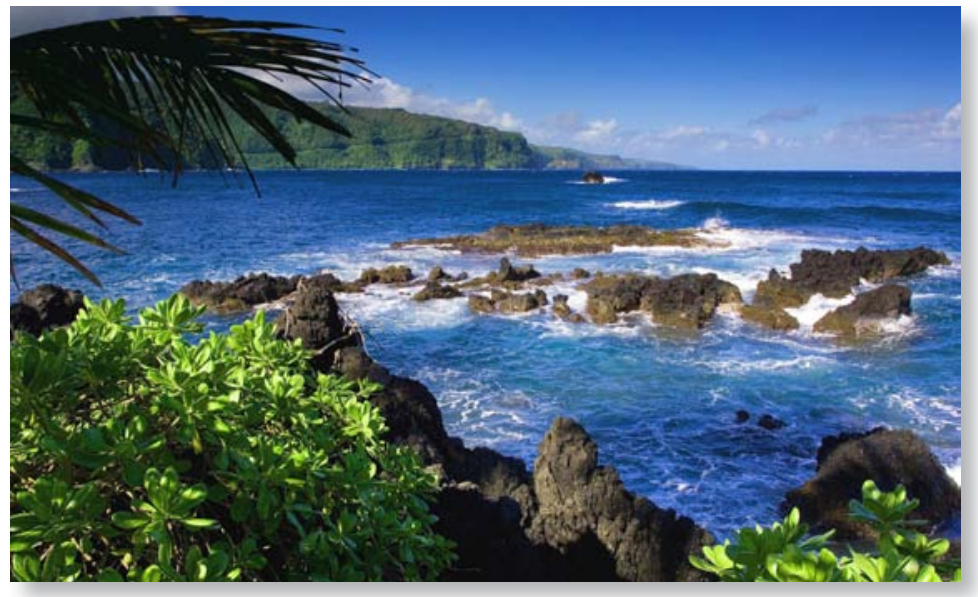

